\title{
Progression of early postnatal retinal pathology in a mouse model of neuronal ceroid lipofuscinosis
}

'Department of Ophthalmology, Ross Eye Institute, Physiology and Biophysics, University at Buffalo-SUNY, Buffalo, NY, USA

${ }^{2}$ Ophthalmology, University of Rochester, Rochester, NY, USA

${ }^{3}$ Institute for Basic Research, Staten Island, NY, USA

Correspondence: GM Seigel,

University at Buffalo, Department

Ophthalmology,

Ross Eye Institute,

Physiology and Biophysics, 3435 Main Street, Sherman 124, Buffalo, NY 14214,

USA

Tel.: + 1716829 2157;

Fax: + 17168292344 .

E-mail: gseige|@

frontiernet.net

Received: 15 July 2004 Accepted: 4 October 2004 Published online: 26 November 2004

\begin{abstract}
Purpose Accumulation of autofluorescent storage material in the CNS is a hallmark of neuronal ceroid lipofuscinosis (NCL, Batten disease). Since the retina is generally the first CNS target affected in NCL and could serve as a means to assess early disease progression as well as potential therapeutic responses, we followed the course of postnatal retinal pathology in tissues from the CLN8 (mnd) mouse model of NCL.

Results Cytoplasmic inclusions in the retinal ganglion cell (RGC) layer were shown by periodic acid schiff stain by P7. TUNEL measurements of cell death became significant at P21 $(P<0.001)$ with most cell death occurring in the photoreceptor layer. Significant autofluorescence and RGC hypertrophy were evident in mnd mice at $\mathrm{PO}$, prior to eye opening or significant cell death. Conclusion An increased understanding of the timing, location, and characteristic retinal pathologies of Batten disease may lead to diagnostic and therapeutic advances in the clinical setting.

Eye (2005) 19, 1306-1312. doi:10.1038/sj.eye.6701770; published online 26 November 2004
\end{abstract}

Keywords: lipofuscinosis; retinal degeneration; apoptosis; pathology

\section{Introduction}

Neuronal ceroid lipofuscinosis (NCL, Batten disease) is a progressive, degenerative disease of the central nervous system. Traditionally, NCL diseases, based on the onset and clinical course of the disease, have been divided into several subtypes: infantile, CLN1 (Haltia-
GM Seigel', J Wagner², A Wronska³ , L Campbell', $\mathrm{W} \mathrm{Ju}^{3}$ and $\mathrm{N}$ Zhong ${ }^{3}$
Santavuori disease); late infantile CLN2 (JanskyBielschowsky disease); juvenile CLN3 (Batten disease or Spielmeyer-Sjogren disease); and the rare, adult, CLN4 (Kufs disease). The newest member of the NCL family is a Finnish variant associated with the CLN8 gene. ${ }^{1,27}$ Progressive epilepsy with mental retardation (EPMR), also known as Northern epilepsy, was recently identified as one of the NCL family by accumulation of autofluorescent, intracytoplasmic storage material in CNS neurons. ${ }^{1,2}$ Electron microscopic observation revealed curvilinear-like profiles and granular storage material comprising mostly subunit $\mathrm{c}$ of mitochondrial ATP synthase. ${ }^{1}$ The CLN8 gene underlying EPMR was identified by positional cloning, ${ }^{2}$ and found to encode a novel protein of 286 amino acids and an estimated molecular weight of $30 \mathrm{kDa}$. Mutations in the CLN8 gene were found to be associated with not only EPMR patients but also the mnd mouse, a model of motor neuron degeneration. ${ }^{2,26}$ The CLN8 gene product has been localized to the endoplasmic reticulum (ER), ${ }^{3}$ with several putative functions proposed, including protein translocation across ER membranes, channel formation, or other activities in the early secretory pathway.

Significant photoreceptor degeneration has been demonstrated in the mnd mouse. ${ }^{4,26}$ However, little has been published on this model, with regard to retinal pathology ${ }^{5}$ or early postnatal events. The CLN8 (mnd) mouse, with an early disease onset and severe retinal degeneration contrasts with the late-onset, mild retinal pathology that we previously characterized in the CLN3 knockout mouse. ${ }^{6}$ The present study focuses on the earliest postnatal changes in the $m n d / m n d$ mouse retina, with specific emphasis on retinal ganglion cell (RGC) pathologies. Since the retina is generally the first CNS target affected in Batten disease 
and could serve as a convenient means to assess the early progression of the disease as well as potential therapeutic responses, we followed a time course of retinal pathology in postnatal tissues from the mnd/mnd mouse.

\section{Methods}

\section{Animals}

All animal experimentation was accomplished in accordance with the Association for Research in the Vision and Ophthalmology statement on the use of animals in research. All studies described in this report were performed on mnd/mnd (AK.B6-Cln8mnd/J) and control mice (Jackson Labs, Bar harbor, ME, USA) aged P0-2 months. Control mice were C57BL/6J (stock No. 000664), as recommended by the Jackson Laboratory. The animals were anaesthetized with $0.2 \mathrm{~cm}^{3}$ of $50 \mathrm{mg} / \mathrm{ml}$ pentobarbital. The mice up to P7 were decapitated, the heads were fixed in $2 \% \mathrm{PFA}$ at $4{ }^{\circ} \mathrm{C}$ for $24 \mathrm{~h}$, then the eyes were removed and stored in $70 \%$ ethanol. Mice older than P7 were perfused transcardially with PBS (to flush out blood) and then with $2 \%$ PFA. Eyes were removed and postfixed in $2 \%$ PFA at $4{ }^{\circ} \mathrm{C}$ for $24 \mathrm{~h}$, and then transferred to $70 \%$ ethanol. A total of 48 eyes were harvested from 24 animals. A developmental series of mnd mice (P0, P2, P4, P7, P14, P21, 1 mo., 2 mo.) and controls were prepared for light microscopy. For each time point, there were six mnd/mnd eyes and six control eyes. Whole eyes were fixed for $24 \mathrm{~h}$ after enucleation, and then embedded in paraffin and cut into $4 \mu \mathrm{m}$ sections (three sections per slide) for further histological analysis.

\section{Periodic acid-Schiff}

Slides were hydrated through Propar (a less toxic alternative to xylene) (Anatech LTD, Battle Creek, MI, USA) and graded ethanols, rinsed in water, and incubated in $0.5 \%$ Periodic acid for $8 \mathrm{~min}$. After three rinses in water, the slides were incubated in warmed Schiff's reagent (Surgipath Medical Ind. Inc., Richmond, IL, USA) for $10 \mathrm{~min}$. Following a water rinse, the slides were counterstained with Harris' hematoxylin for $5 \mathrm{~min}$. The slides were immersed in running water for $1 \mathrm{~min}$, Clarifier \#2 (Richard-Allen Medical, Richland, MI, USA) for $30 \mathrm{~s}$, and then water for $1 \mathrm{~min}$, followed by 10 sequential rinses in a weak ammonia solution. The slides were dehydrated and covered with Permount.

\section{Terminal deoxynucelotidyltransferase-mediated UTP end labelling (TUNEL) in situ analysis}

Tissue sections were deparaffinized in xylene and graded alcohols. Fragmented DNA was labelled using the
Apoptag Plus Apoptosis Detection Kit (Intergen, Inc., Purchase, NY, USA), and developed with diaminobenzidine reaction product. DAB colour development was allowed to proceed for $5 \mathrm{~min}$. Tissue sections were counterstained in methyl green and coverslipped for microscopic observation. TUNEL reactive cells were counted per random $\times 40$ field in a masked fashion (five counts per section, three sections per slide). Results were analysed and graphed using Prism 4 software (Graphpad, Inc., San Diego, CA, USA). Bonferroni post-tests were used to determine significance and $P$-values. Results were considered significant if $P<0.05$.

\section{Fluorescence microscopy}

After TUNEL analysis was completed, tissue sections were visualized by fluorescence microscopy with an Olympus Provis AX70 microscope. The standard method of visualizing autofluorescence was accomplished as we have carried out previously ${ }^{6}$ with the $U$ cube (excitation 360-370, barrier filter BA 420, dichroic mirror DM400).

These settings were appropriate for detecting the autofluorescence typical of NCL tissues.

\section{Morphometry}

RGC dimensions (10 samplings per three sections of each timepoint) were measured using Neurolucida (Microbrightfield, Inc. Williston, VT, USA). Cells for measurement were chosen randomly from the more central regions of the retina. Circular tracings were used to measure ganglion cell diameter, circumference, area, and volume throughout the postnatal timecourse of this study. Results were analysed and graphed using Prism 4 software. Bonferroni post-tests were used to determine significance and $P$-values. Results were considered significant if $P<0.05$.

\section{Results}

\section{Retinal autofluorescence is evident in mnd mice at P0}

Ocular sections from the developmental series of $\mathrm{mnd} /$ mnd mice were visualized by fluorescence microscopy at $410 \mathrm{~nm}$ and compared with controls. Examples of P0, P4, and 2 months are shown in Figure 1. There was an overall higher level of autofluorescence throughout the retinal tissue, as well as particular cells that were especially bright, especially in the RGC. These are indicated by arrows in Figure 1. Increased autofluorescence was evident in mnd/mnd mice throughout the postnatal time course of this study. 

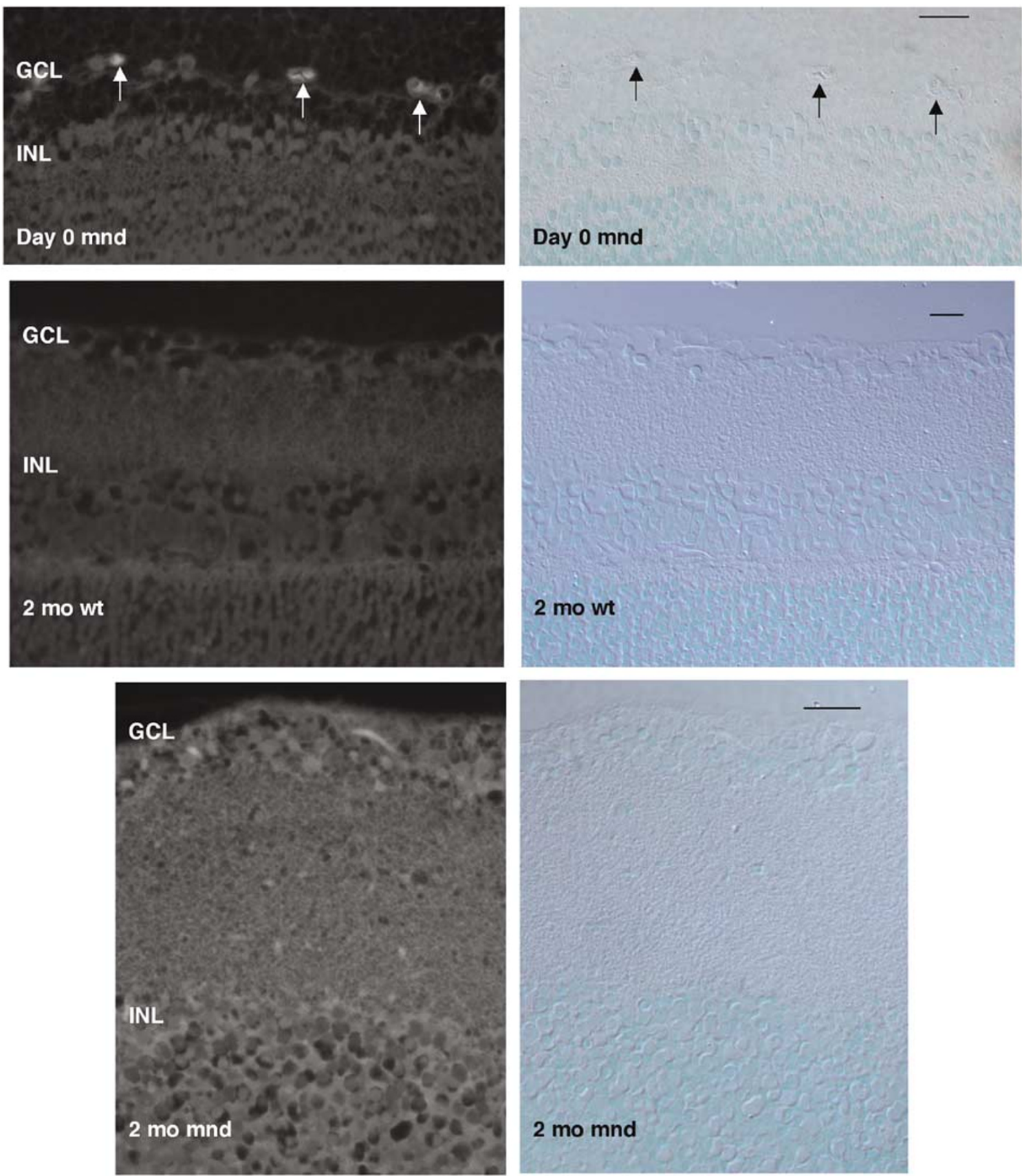

Figure 1 Retinal autofluorescence is evident in mnd mice at P0. Ocular sections from the developmental series of $m n d$ mice were visualized by fluorescence microscopy and compared with controls. Exposure times were kept constant for all fluorescent photomicrographs. Examples of P0 and 2 months are shown. The arrows indicate cells that are particularly autofluorescent. Both brightfield and fluorescent fields are shown. Scale bar $=10 \mu \mathrm{m}$.

\section{Significant cell death is evident in mnd retina by P21}

Tissues were stained for TUNEL reactivity to detect DNA fragmentation indicative of cell death. The number of
TUNEL reactive cells was counted per $\times 40$ field. As seen in Figure 2, following a period of developmental cell death in all mice, the number of TUNEL-reactive cells became statistically significant in mnd mouse retina by 

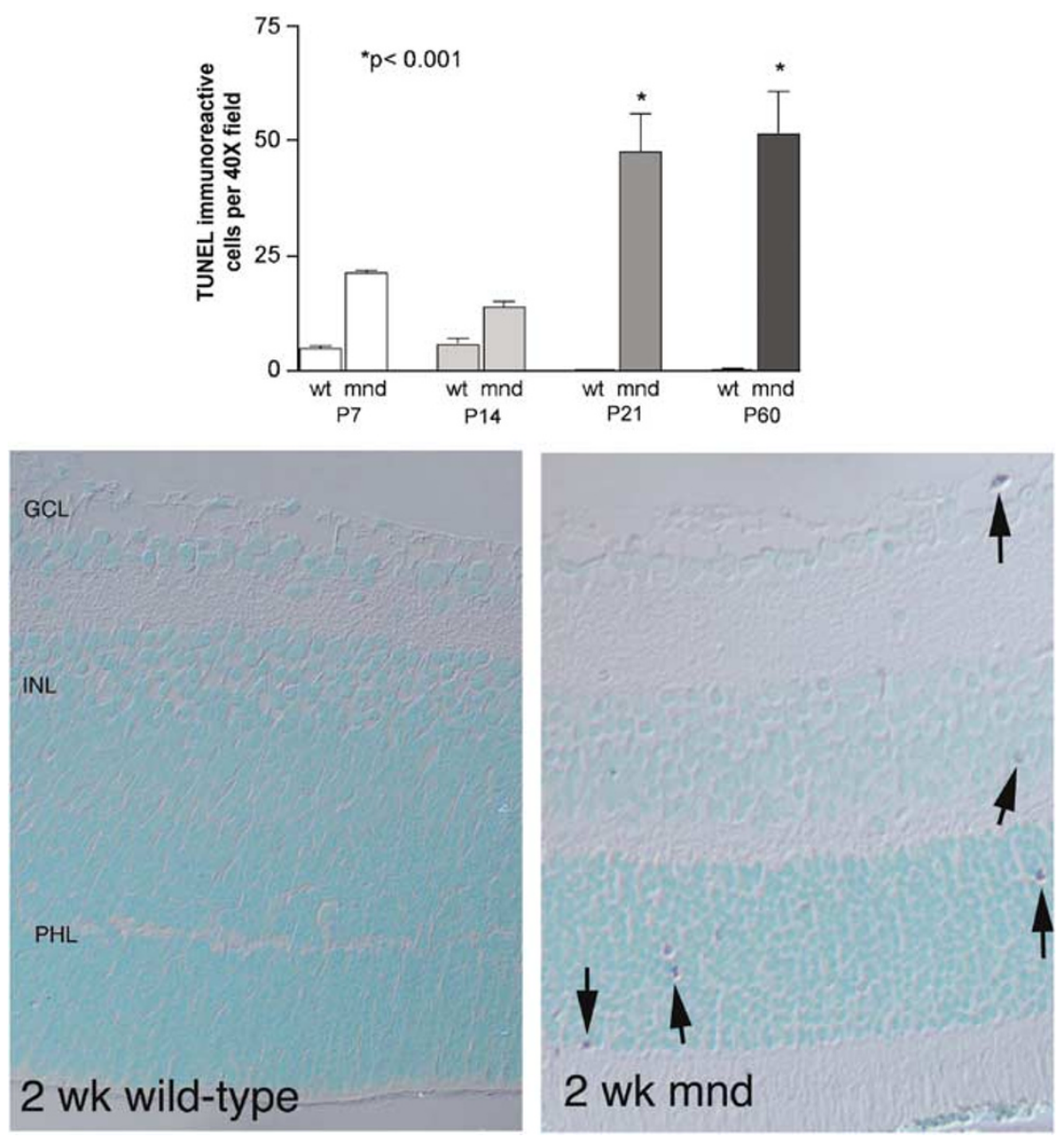

Figure 2 Significant cell death is evident in mnd retina by P21. Tissues were stained for TUNEL reactivity to detect DNA fragmentation indicative of cell death. The number of TUNEL reactive cells was counted per $\times 40$ field. As seen in Panel a, the number of TUNEL-reactive cells became statistically significant by P21. Examples of TUNEL histology from P14 mouse retina are shown in Panels b and c. Positive cells are seen in the PHL and GCL of the mnd mouse (arrows), but not in controls. Scale bar $=10 \mu \mathrm{m}$.

P21. The majority of TUNEL-positive cells were seen in the photoreceptor layer $(\mathrm{Ph})$, as seen in the representative photomicrographs of P14 tissues in Figure 2(b and c).

\section{RGC layer pathology in mnd mice}

Carbohydrate storage material was seen with PAS (periodic acid-Schiff) stain in RGCs. Examples of this are shown in 2-week-old mnd/mnd mice (Figure 3). In contrast, control mice did not demonstrate PAScontaining material (Figure 3).

\section{Hypertrophy of mnd RGCs throughout postnatal life}

In the histological observations of Figure 3, it appeared that mnd RGCs were engorged with storage material that might affect cell size. Therefore, we measured random RGC dimensions in the central retina using Neurolucida software. RGCs of mnd mice were significantly enlarged, as measured by diameter, circumference, area, and volume, throughout the postnatal time course of this study, as seen in Figure 4.

\section{Discussion}

This study describes very early postnatal pathological changes in retinal tissues of $m n d / m n d$ mice. Previous studies examined $\mathrm{mnd} / \mathrm{mnd}$ retinal tissue as early as P10 and found no differences in parameters such as the number of pyknotic nuclei, thickness of the outer nuclear layer, or photoreceptor inner/outer segment lengths until P15. ${ }^{4}$ The most striking observations in the present study are the significant increases in autofluorescence and RGC size as early as P0, prior to eye opening in the mouse (P8-10). RGCs are the first retinal cell type to mature, between E11 and P0 see review ${ }^{7}$ ). It is reasonable to predict that CLN8-mediated pathological changes may 

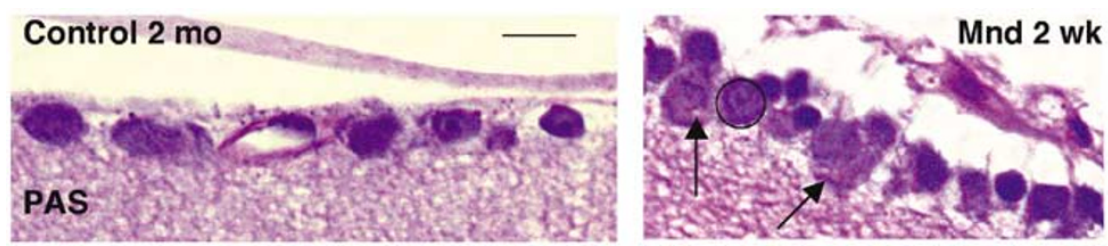

Figure 3 Retinal ganglion cell pathology of $m n d$ mice. Carbohydrate storage material is seen with PAS (periodic acid-schiff) stain in engorged retinal ganglion cells of 2-week-old mnd mice (arrows). In contrast, control mice do not demonstrate PAS-containing material. A 2-month-old control mouse is shown in comparison (oldest timepoint in our study). Circle/oval indicates an example of how morphometric measurements were taken for Figure 4 . Scale bar $=10 \mu \mathrm{m}$.

Diameter

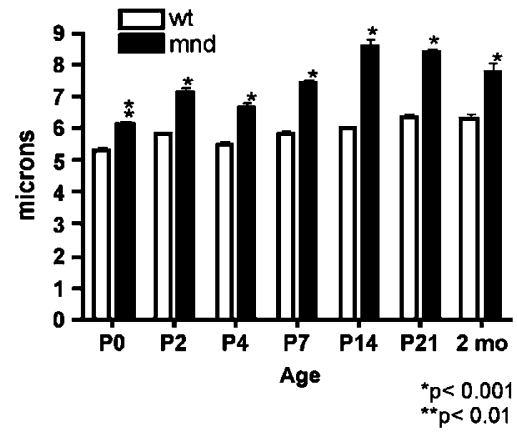

Area

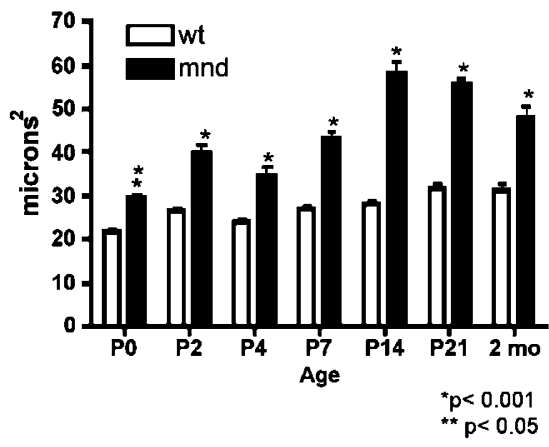

Circumference

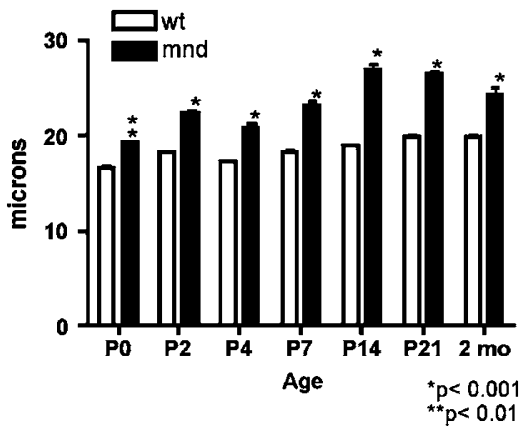

Volume

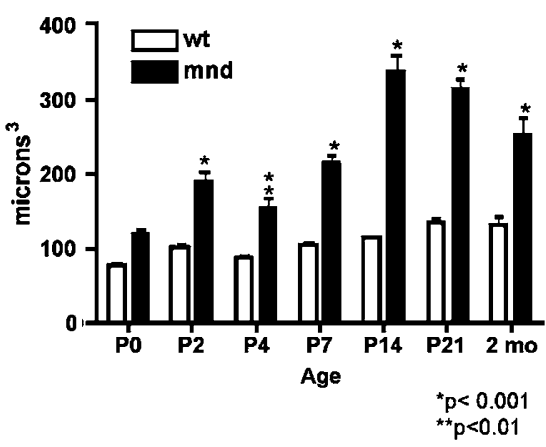

Figure 4 Enlargement of $m n d$ retinal ganglion cells throughout postnatal life. Retinal ganglion cells of mnd mice were significantly enlarged, as measured by diameter, circumference, area, and volume, throughout the postnatal time course of this study. Random measurements of retinal ganglion cell dimensions (10 samplings per time point) were taken in central regions of the retina using Neurolucida (Microbrightfield, Inc., Williston, VT, USA).

occur prior to birth. Future examinations of embryonic $m n d / m n d$ mice will address this issue.

\section{RGC hypertrophy}

RGCs are the only cells of the retina with a direct connection to the brain. RGC hypertrophy in the present study mirrors changes seen in $m n d / m n d$ mouse brain, in which several subpopulations of interneurons were found to be significantly larger in the $m n d / m n d$ mouse than in controls. ${ }^{8}$ Most retinal degenerative processes affect either photoreceptors or RGCs. The mnd/mnd mouse represents a special phenotype in which both photoreceptors and RGCs are degenerating, along with other CNS neurons. No mechanistic link between the cell types has been clarified, but may represent common biochemical alterations of the $m n d / m n d$ phenotype that lead to CNS degeneration.

Hypertrophy of RGCs has been described qualitatively in NCLs. In our previous study of the less degenerative CLN3 mouse, RGCs were engorged with PAS-positive inclusions. ${ }^{6}$ RGCs of ovine NCL (CLN6) were also described as enlarged. ${ }^{9}$ In a case study of adult-onset human NCL (CLN4), RGCs exhibited signs of storage material. ${ }^{10}$ It appears that RGC soma enlargement may be accompanied by RGC axonal changes through the 
optic nerve as well. In a pooled sample of 4-10-monthold mice, diminished axon density in the optic nerve was reported in the aforementioned CLN3 model of $\mathrm{NCL}^{11}$ (D. Calkins, personal communication). Thus, RGC parameters, such as cell soma size and axon density, appear to be pathologies common to multiple NCL models.

In $m n d / m n d$ mice, we found a peak of RGC cell size at P14, followed by a drop-off in size through 2 months. This peak in RGC cell size immediately preceded the peak in the number of TUNEL-reactive cells and extensive cell death seen at P21. Taken together, one simple explanation for these findings is that the largest RGCs apoptose after P14, leaving the somewhat smaller RGCs behind that were measured at P21 and beyond. Our findings that cell death becomes most pronounced at P21, especially in the photoreceptor layer of $m n d / m n d$ mice, corresponds well with previous studies in which photoreceptor layer thinning accelerates by $\mathrm{P} 25 .{ }^{4}$ One possible mechanism is that the transmembrane CLN8 protein plays a role as an antiapoptotic agent, as demonstrated for the CLN3 protein. ${ }^{12}$ Further studies are necessary to confirm a similar function for CLN8 in the protection of neurons from apoptotic cell death.

\section{Storage material in RGCs}

Batten disease is characterized by the accumulation of autofluorescent hydrophobic material in the cytoplasm of neurons, and to a lesser extent, other cell types. Based on their composition and ultrastructure, these deposits are presumed to be localized to the lysosomes. ${ }^{13}$ The electron-dense storage material in the juvenile form of Batten disease has a fingerprint-like appearance. ${ }^{14,15}$ Furthermore, protein sequencing and immunological studies have revealed that subunit $\mathrm{c}$ of the mitochondrial ATP synthase is the major component of the storage material in Batten disease. ${ }^{16,17}$ Autofluorescent storage material is readily visible in $m n d / m n d$ mice at our earliest postnatal time point, P0, with likely involvement during embryonic development.

\section{Metabolic changes in $\mathrm{mnd} / \mathrm{mnd}$ mice}

Important biochemical changes underlying degeneration of the central nervous system in NCLs are still being identified. Griffin and colleagues ${ }^{18}$ found CLN8-induced vitamin $\mathrm{E}$ deficiency, coupled with increases in glutamate and $N$-acetyl-L-aspartate, and decreases in creatine, glutamine, aspartate, GABA in the CLN8 mouse brain. Diet supplementation with vitamin $\mathrm{E}$ was not able to overcome these metabolic abnormalities and did not prevent neurodegeneration in the CLN8 brain. Those results as well as other studies in the CLN8 liver ${ }^{19}$ and
CLN3 lymphoblasts ${ }^{20}$ point to alterations in mitochondrial activity, which fits well with the possibility of oxidative stress mismanagement in this disease. ${ }^{30}$ Mitochondrial biogenesis occurs within the cell bodies of RGCs, followed by transport along the axons leading to the optic nerve. Mitochondrial functions including oxidative stress and mitochondrial inner membrane potential affect the opening of the mitochondrial permeability transition pore through which proapoptotic factors reach the cytosol. ${ }^{21-23}$ These mitochondrial mechanisms appear to play a role in RGC death in axotomy ${ }^{24}$ and may also pertain to mitochondrial diseases that specifically affect RGCs, such as Leber's hereditary optic neuropathy. Further studies at the proteomic and genetic levels will seek to address the neurodegenerative processes inherent in NCLs which lead to such early and devastating consequences in the CNS.

\section{Acknowledgements}

This work was supported in part by NIH/NINDS R21NS042806 (NZ) and Children's Brain Diseases Foundation (NZ).

\section{References}

1 Herva R, Tynela J, Hirvasniemi A, Syrjakallio-Ylitalo M, Haltia M. Northern epilepsy: a novel form of neuronal ceroid lipofuscinosis. Brain Pathol 2000; 10: 215-222.

2 Ranta S, Zhang Y, Ross B, Lonka L, Takkunen E, Messer A et al. The neuronal ceroid lipofuscinoses in human EPMR and mnd mutant mice are associated with mutations in CLN8. Nat Genet 1999; 23(2): 233-236.

3 Lonka L, Kyttala A, Ranta S, Jalanko A, Lehesjoki AE. The neuronal ceroid lipofuscinosis CLN8 membrane protein is a resident of the endoplasmic reticulum. Hum Mol Genetics 2000; 9(11): 1691-1697.

4 Messer A, Plummer J, Wong V, Lavail M. Retinal degeneration in motor neuron degeneration (mnd) mouse. Exp Eye Res 1993; 57: 637-641.

5 Chang B, Bronson RT, Hawes NL, Roderick TH, Peng C, Hageman GS et al. Retinal degeneration in motor neuron degeneration: a mouse model of ceroid lipofuscinosis. Invest Ophthalmol Vis Sci 1994; 35(3): 1071-1076.

6 Seigel GM, Lotery A, Kummer A, Bernard D, Greene NDE, Turmaine $\mathrm{M}$ et al. Retinal pathology and function in a cln3knockout mouse model of juvenile ceroid lipofuscinosis (Batten Disease). Mol Cell Neurosci 2002; 19: 515-527.

7 Isenmann S, Kretza A, Cellerino A. Molecular determinants of retinal ganglion cell development, survival, and regeneration. Prog Ret Eye Res 2003; 22: 483-543.

8 Cooper JD, Messer A, Feng AK, Chua-Couzens J, Mobley WC. Apparent loss and hypertrophy of interneurons in a mouse model of neuronal ceroid lipofuscinosis: evidence for partial response to insulin-like growth factor- 1 treatment. J Neurosci 1999; 19(7): 2556-2567. 
9 Murnane RD, Prieur DJ, Ahern-Rindell AJ, Parish SM, Collier LL. The lesions of an ovine lysosomal storage disease. Initial characterization. Am J Pathol 1989; 134(2): 263-270.

10 Martin JJ, Libert J, Ceuterick C. Ultrastructure of brain and retina in Kufs' disease (adult type-ceroid-lipofuscinosis). Clin Neuropathol 1987; 6(6): 231-235.

11 Sappington RM, Pearce DA, Calkins DJ. Optic nerve degeneration in a murine model of juvenile ceroid lipofuscinosis. Invest Ophthalmol Vis Sci 2003; 44(9): 3725-3731.

12 Persaud-Sawin DANW, VanDongen A, Boustany R. Motifs within the CLN3 protein: modulation of cell growth rates and apoptosis. Hum Mol Genet 2002; 11(18): 2129-2142.

13 Carpenter S. Morphological diagnosis and misdiagnosis in Batten-kufs disease. Am J Med Genet Suppl 1988; 5: 85-91.

14 Goebel HH, Gullotta F, Bajanowski T, Hansen FJ, Braak H. Pigment variant of neuronal ceroid-lipofuscinosis. Am J Med Genet 1995; 57: 155-159.

15 Rapola J. Neuronal ceroid-lipofuscinoses in childhood. Perspect Pediatr Pathol 1993; 17: 7-44.

16 Hall NA, Lake BD, Dewji NN, Patrick AD. Lysosomal storage of subunit $\mathrm{c}$ of mitochondrial ATP synthase in Batten's disease (ceroid-lipofuscinosis). Biochem J 1991; 275: 269-272.

17 Palmer DN, Fearnley IM, Walker JE, Hall NA, Lake BD, Wolfe LS et al. Mitochondrial ATP synthase subunit c storage in the ceroid-lipofuscinoses (Batten disease). Am J Med Genet 1992; 42: 561-567.

18 Griffin JL, Muller R, Woograsingh R, Jowatt V, Hindmarsh A, Nicholson JK et al. Vitamin E deficiency and metabolic defects in neuronal ceroid lipofuscinosis described by bioinformatics. Physiol Genomics 2002; 11: 195-203.

19 Vance JE, Stone SJ, Faust JR. Abnormalities in mitochondriaassociated membranes and phospholipid biosynthetic enzymes in the mnd/mnd mouse model of neuronal ceroid lipofuscinosis. Biochim Biophys Acta 1997; 1344: 286-299.

20 Boriack RL, Cortinas E, Bennett MJ. Mitochondrial damage results in a reversible increase in lysosomal storage material in lymphoblasts from patients with juvenile neuronal ceroid-lipofuscinosis (Batten Disease). Am J Med Genet 1995; 57(2): 301-303.

21 Zoratti M, Szabo I. The mitochondrial permeability transition. Biochim Biophys Acta 1995; 1241: 139-176.

22 Green DR, Reed JC. Mitochondria and apoptosis. Science 1998; 281: 1309-1312.

23 Carellia V, Ross-Cisnerosa FN, Saduna AA. Mitochondrial dysfunction as a cause of optic neuropathies. Prog Ret Eye Res 2004; 23: 53-89.

24 Vrabec JP, Lieven CJ, Levin LA. Cell-type-specific opening of the retinal ganglion cell mitochondrial permeability transition pore. Invest Ophthalmol Vis Sci 2003; 44(6): 2774-2782.

25 Cai J, Nelson K, Wu M, Sternberg P, Jones DP. Oxidative damage and protection of the RPE. Prog Ret Eye Res 2000; 19(2): 205-221.

26 Messer A, Manley K, Plummer J. An early-onset strain of motor neuron degeneration (mnd) mutant mice. Mol Genet Metab 1999; 66: 393-397.

27 Ranta S, Lehesjoki AE. Northern epilepsy, a new member of the NCL family. Neurol Sci 2000; 21: S43-S47.

28 Sandbach J, Coscun P, Grossniklaus H, Kokoszka JE, Newman NJ, Wallace DC. Ocular pathology in mitochondrial superoxide dismutase (Sod2)-deficient mice. Invest Ophthalmol Vis Sci 2001; 42(10): 2173-2178.

29 Shamsi F, Boulton M. Inhibition of RPE lysosomal and antioxidant activity by the age pigment lipofuscin. Invest Ophthalmol Vis Sci 2001; 42(12): 3041-3046.

30 Sundelin S, Nilsson S. Lipofuscin-formation in retinal pigment epithelial cells is reduced by anti-oxidants. Free Rad Biol Med 2001; 31(2): 217-225. 